\title{
A New Finite-Time Bounded Control of Stochastic Itô Systems with $(x, u, v)$-Dependent Noise: Different Quadratic Function Approach
}

\author{
Zhiguo Yan' ${ }^{1}$ and Zhongwei Lin ${ }^{2}$ \\ ${ }^{1}$ School of Electrical Engineering and Automation, Qilu University of Technology, Jinan 250353, China \\ ${ }^{2}$ State Key Laboratory of Alternate Electrical Power System with Renewable Energy Sources, School of Control and \\ Computer Engineering, North China Electric Power University, Beijing 102206, China
}

Correspondence should be addressed to Zhiguo Yan; yanzg500@sina.com

Received 24 November 2013; Accepted 11 January 2014; Published 24 March 2014

Academic Editor: Hui Zhang

Copyright (C) 2014 Z. Yan and Z. Lin. This is an open access article distributed under the Creative Commons Attribution License, which permits unrestricted use, distribution, and reproduction in any medium, provided the original work is properly cited.

\begin{abstract}
This paper addresses the finite-time bounded control problem of linear stochastic systems with state, control input, and external disturbance-dependent noise $((x, u, v)$-dependent noise for short). The notion of finite-time boundedness of linear stochastic systems is first introduced. Then a different quadratic function approach is proposed to give a sufficient condition for finite-time boundedness of such a class of systems, and its superiority to common quadratic approach is shown. Moreover, the finite-time bounded controller design problem is studied and two sufficient conditions for the existence of state and output feedback controllers are presented in terms of nonlinear matrix inequalities. An algorithm is given for solving the obtained nonlinear matrix inequalities. Finally, an example is employed to illustrate the effectiveness of our obtained results.
\end{abstract}

\section{Introduction}

It is well known that finite-time control has become one of the important robust control methods, which has been studied extensively both in theory and practical applications; see linear systems [1-9], nonlinear systems [10-12], and the inpress book [13]. Recently, based on analysis on some practical problems, [14] introduced a new finite-time stability for linear stochastic Itô systems with state and control-dependent noise. Roughly speaking, a stochastic Itô system is said to be finitetime stable if, given a bound on the initial state of the system, its state trajectories do not exceed an upper bound $c_{2}$ and are not less than a lower bound $c_{1}\left(c_{1}<c_{2}\right)$ in the mean square sense during a specific time interval.

On the other hand, the model of stochastic Itô systems with state, control input, and external disturbance dependent-noise $((x, u, v)$-dependent noise for short) is more general than stochastic Itô systems with state and control input-dependent noise $((x, u)$-dependent noise for short). For this class of model, some results have been obtained.
For example, $[15,16]$ studied the finite/infinite horizon mixed $H_{2} / H_{\infty}$ control problem for the Itô-type nonlinear stochastic systems with $(x, u, v)$-dependent noise, respectively.

Motivated by aforementioned discussions, we extend the results in [14] to stochastic Itô systems with $(x, u, v)$ dependent noise. Here, we consider finite-time stochastic boundedness and finite-time bounded control problems for such class of systems. More precisely, a system is said to be finite-time bounded if, given a bound both on the initial state of the system and the disturbance input, the state trajectories of the system do not exceed an upper bound $c_{2}$ and are not less than a lower bound $c_{1}\left(c_{1}<c_{2}\right)$ in the mean square sense during a prespecified time interval for all admissible disturbances. By stochastic analysis technology, Gronwall's inequality, and matrix transformation, a finite-time stochastic boundedness criterion and some sufficient conditions for the existence of finite-time bounded controller are derived. The contributions of this paper lie in the following two aspects: (1) a new concept of finite-time stochastic boundedness is introduced, which generalizes the finite time stochastic stability in 
[14] to stochastic Itô systems with $(x, u, v)$-dependent noise and (2) a different quadratic function approach is introduced and its superiority to common quadratic function approach is shown. By different quadratic function approach, two new conditions for the existence of state and output feedback finite-time bounded controller are obtained.

The paper is organized as follows. In Section 2, a concept of finite-time stochastic boundedness and some preliminaries are presented. Section 3 provides a sufficient condition for finite-time stochastic boundedness. In Section 4, state and output finite-time bounded controllers are given, respectively. Section 5 employs an example to illustrate the results of the paper. Section 6 gives the conclusion.

Notation. $A^{T}$ is a transpose of a matrix or vector $A$. $A>0(A \geq 0): A$ is positive definite (positive semidefinite) symmetric matrix. $L_{\mathscr{F}}^{2}\left(\mathbf{R}_{+}, \mathbf{R}^{l}\right)$ is a space of nonanticipative stochastic process $y(t) \in \mathbf{R}^{l}$ with respect to an increasing $\sigma$ algebra $\mathscr{F}_{t}(t \geq 0)$ satisfying $\mathbb{E} \int_{0}^{\infty}\|y(t)\|^{2} d t<\infty$. $\mathbb{E}[\cdot]$ stands for the mathematical expectation operator with respect to the given probability measure. $I_{n \times n}$ is $n \times n$ identity matrix. $\operatorname{tr}(A)$ is trace of a matrix $A$. $\lambda_{\max }(A)\left(\lambda_{\min }(A)\right)$ is the maximum (minimum) eigenvalue of a real matrix $A$.

\section{Preliminaries and Problem Statement}

Consider the following linear time-invariant stochastic Itô system with $(x, u, v)$-dependent noise:

$$
\begin{gathered}
d x(t)=(A x(t)+B u(t)+G v(t)) d t \\
+\left(A_{1} x(t)+B_{1} u(t)+G_{1} v(t)\right) d w(t), \\
y(t)=C x(t), \quad x(0)=x_{0} \in \mathbf{R}^{n},
\end{gathered}
$$

where $x(t) \in \mathbf{R}^{n}, u(t) \in \mathbf{R}^{m}, v(t) \in \mathbf{R}^{l}$, and $y(t) \in \mathbf{R}^{p}$ are called the system state, control input, exogenous disturbance, and measurement output, respectively. $A, A_{1} \in \mathbf{R}^{n \times n}, B, B_{1} \in$ $\mathbf{R}^{n \times m}, G, G_{1} \in \mathbf{R}^{n \times l}$, and $C \in \mathbf{R}^{p \times n}$ are constant matrices. $x_{0}$ is the initial state. Without loss of generality, throughout this paper, we assume $w(t)$ to be one-dimensional standard Wiener process defined on the probability space $\left(\Omega, \mathscr{F}, \mathscr{F}_{t}\right.$, $P$ ) with $\mathscr{F}_{t}=\sigma\{w(s): 0 \leq s \leq t\}$.

To illustrate clearly the concept of finite-time stochastic boundedness presented below, we first introduce finite-time stochastic stability from [14].

Definition 1. Given positive real scalars $c_{1}, c_{2}, c_{3}, c_{4}, T$ with $0<c_{1}<c_{3}<c_{4}<c_{2}$, and a positive definite matrix $R$, the following linear stochastic system

$$
\begin{gathered}
d x(t)=A x(t) d t+A_{1} x(t) d w(t) \\
x(0)=x_{0},
\end{gathered}
$$

is said to be finite-time stochastically stable with respect to $\left(c_{1}, c_{2}, c_{3}, c_{4}, T, R\right)$, if

$$
\begin{aligned}
c_{3} \leq & \mathbb{E}\left[x^{T}(0) R x(0)\right] \leq c_{4} \\
& \Longrightarrow c_{1}<\mathbb{E}\left[x^{T}(t) R x(t)\right]<c_{2}, \quad \forall t \in[0, T] .
\end{aligned}
$$

Based on Definition 1, a new concept of finite-time stochastic boundedness for linear stochastic Itô systems is introduced.

Definition 2. Given some positive scalars $c_{1}, c_{2}, c_{3}, c_{4}$, $T$ with $0<c_{1}<c_{3}<c_{4}<c_{2}$, a positive definite matrix $R$, and a class of exogenous signals $\mathscr{W}$, the following linear stochastic system

$$
\begin{gathered}
d x(t)=(A x(t)+G v(t)) d t \\
+\left(A_{1} x(t)+G_{1} v(t)\right) d w(t) \\
x(0)=x_{0}
\end{gathered}
$$

is said to be finite-time stochastically bounded with respect to $\left(c_{1}, c_{2}, c_{3}, c_{4}, \mathscr{W}, T, R\right)$, if

$$
\begin{aligned}
c_{3} \leq & \mathbb{E}\left[x^{T}(0) R x(0)\right] \leq c_{4} \\
& \Longrightarrow c_{1}<\mathbb{E}\left[x^{T}(t) R x(t)\right]<c_{2}, \quad \forall t \in[0, T],
\end{aligned}
$$

for all $v(\cdot) \in \mathscr{W}$.

Remark 3. Definition 2 is more general than Definition 1, which concerns the behavior of the state in the presence of both given initial conditions and external disturbance.

Remark 4. It is clear that finite-time stochastic boundedness implies finite-time stochastic stability, but the converse is not true.

In the next assumption, we characterize a class of signals $\mathscr{W}$ considered in this paper.

Assumption 5. The class $\mathscr{W}$ is defined as follows:

$$
\begin{aligned}
\mathscr{W}=\{v(t): d v(t) & =H v(t) d t+H_{1} v(t) d w(t), \\
& \left.v(0)=v_{0} \in \mathbf{R}^{l}, v_{0}^{T} R_{1} v_{0} \leq h_{1}, \mathbb{E}\left[v^{T}(t) R_{1} v(t)\right]<h\right\},
\end{aligned}
$$

where $H, H_{1}$, and $R_{1} \geq 0$ are constant matrices and $h$ and $h_{1}$ are any given positive scalars.

Remark 6. In Assumption 5, $h$ and $h_{1}$ are any given positive scalars, so $\mathscr{W}$ actually includes a big class of signals.

Before proceeding further, we give some lemmas which will be used in the next section.

Lemma 7 (Itô-type formula). For given $V(x) \in C^{2}\left(\mathbf{R}^{n}\right)$, associated with the following stochastic system

$$
d x(t)=f(x) d t+g(x) d w(t),
$$

the infinitesimal generator operator is defined by

$$
\mathscr{L} V(x)=\frac{\partial V(x)}{\partial x} f(x)+\frac{1}{2} \operatorname{Tr}\left[g^{T}(x) \frac{\partial^{2} V(x)}{\partial x^{2}} g(x)\right]
$$


Lemma 8 (Gronwall Inequality). Let $\theta(t)$ be a nonnegative function such that

$$
\theta(t) \leq a+b \int_{0}^{t} \theta(s) d s, \quad 0 \leq t \leq T
$$

for some constants $a, b \geq 0$; then one has

$$
\theta(t) \leq a \exp (b t), \quad 0 \leq t \leq T .
$$

Lemma 9 (see [14]). Let $\theta(t)$ be a nonnegative function such that

$$
\theta(t) \geq a+b \int_{0}^{t} \theta(s) d s, \quad 0 \leq t \leq T
$$

for some constants $a, b \geq 0$; then one has

$$
\theta(t) \geq a \exp (b t), \quad 0 \leq t \leq T .
$$

\section{Finite-Time Stochastic Boundedness}

This section is dedicated to proposing a different quadratic function approach to the finite-time stochastic boundedness problem of the system (4). The comparison on different quadratic function approach and common quadratic function approach is first given.

In [14], the key approach of obtaining main results is as follows. Let $V\left(S_{\text {state }}\right)$ be a positive quadratic function; then by the following inequalities

$$
\begin{aligned}
& \mathscr{L V}\left(S_{\text {state }}(t)\right)<\alpha V\left(S_{\text {state }}(t)\right), \\
& \mathscr{L} V\left(S_{\text {state }}(t)\right)>\beta V\left(S_{\text {state }}(t)\right),
\end{aligned}
$$

the main results are derived. We call the above approach to be common quadratic function approach, because the quadratic functions in (13) and (14) are the same. But we find that $V\left(S_{\text {state }}(t)\right)$ satisfying (13) may not satisfy (14), which results in the a relatively small range of the option of $V\left(S_{\text {state }}(t)\right)$. So the main results obtained by common quadratic function approach are of conservativeness.

The key idea of different quadratic function approach is as follows. Let $V_{1}\left(S_{\text {state }}(t)\right)$ and $V_{2}\left(S_{\text {state }}(t)\right)$ be a positive quadratic function; then by the following inequalities

$$
\begin{aligned}
& \mathscr{L} V_{1}\left(S_{\text {state }}(t)\right)<\alpha V_{1}\left(S_{\text {state }}(t)\right), \\
& \mathscr{L} V_{2}\left(S_{\text {state }}(t)\right)>\beta V_{2}\left(S_{\text {state }}(t)\right),
\end{aligned}
$$

the main results of this paper will be derived. Because the quadratic functions $V_{1}\left(S_{\text {state }}(t)\right)$ in (15) and $V_{2}\left(S_{\text {state }}(t)\right)$ in (16) are not the same, the main results obtained by this approach are of less conservativeness than the results obtained by common quadratic function approach.

Theorem 10. If there exist $\alpha \geq 0, \beta \geq 0$, symmetric positive definite matrices $Q_{1} \in \mathbf{R}^{n \times n}, Q_{2} \in \mathbf{R}^{l \times l}, Q_{3} \in \mathbf{R}^{l \times l}$ and some scalars $\lambda_{i}>0, i=1,2,3$ such that the following inequalities hold

$$
\left[\begin{array}{ccc}
\widetilde{Q}_{1} A^{T}+A \widetilde{Q}_{1}-\alpha \widetilde{Q}_{1} & G & \widetilde{Q}_{1} A_{1}^{T} \\
* & \Gamma-\alpha \widetilde{Q}_{2} & G_{1}^{T} \\
* & * & -\widetilde{Q}_{1}
\end{array}\right]<0,
$$

$$
\left[\begin{array}{ccc}
\beta \widetilde{Q}_{1}-\widetilde{Q}_{1} A^{T}-A \widetilde{Q}_{1} & G & \widetilde{Q}_{1} A_{1}^{T} \\
* & \beta \widetilde{Q}_{3}-\bar{\Gamma} & G_{1}^{T} \\
* & * & -\widetilde{Q}_{1}
\end{array}\right]<0,
$$

$$
\begin{gathered}
{\left[\begin{array}{cc}
\lambda_{2} h_{1}-c_{2} e^{-\alpha T} & \sqrt{c_{4}} \\
* & -\lambda_{1}
\end{array}\right]<0,} \\
{\left[\begin{array}{cc}
h \lambda_{3}-c_{3} & \sqrt{c_{1}} \\
* & -\lambda_{1}
\end{array}\right]<0,} \\
\lambda_{1} I<Q_{1}<I, \quad 0<Q_{2}<\lambda_{2} I, \\
0<Q_{3}<\lambda_{3} I,
\end{gathered}
$$

then system (4) is finite-time stochastically bounded with respect to $\left(c_{1}, c_{2}, c_{3}, c_{4}, \mathscr{W}, T, R\right)$, where $\widetilde{Q}_{1}=R^{-1 / 2} Q_{1} R^{-1 / 2}$, $\widetilde{Q}_{2}=R_{1}^{1 / 2} Q_{2} R_{1}^{1 / 2}, \widetilde{Q}_{3}=R_{1}^{1 / 2} Q_{3} R_{1}^{1 / 2}, \Gamma=H^{T} \widetilde{Q}_{2}+\widetilde{Q}_{2} H+$ $H_{1}^{T} \widetilde{Q}_{2} H_{1}, \bar{\Gamma}=H^{T} \widetilde{Q}_{3}+\widetilde{Q}_{3} H+H_{1}^{T} \widetilde{Q}_{3} H_{1}$.

Proof.

Step 1. $\mathbb{E}\left[x^{T}(0) R x(0)\right]<c_{4} \Rightarrow \mathbb{E}\left[x^{T}(t) R x(t)\right]<c_{2}$.

Take a quadratic function

$$
V_{1}(x(t), v(t))=x^{T}(t) \widetilde{Q}_{1}^{-1} x(t)+v^{T}(t) \widetilde{Q}_{2} v(t),
$$

where $\widetilde{Q}_{1}=R^{-1 / 2} Q_{1} R^{-1 / 2}, \widetilde{Q}_{2}=R_{1}^{1 / 2} Q_{2} R_{1}^{1 / 2}$ with $Q_{1}>0$, $Q_{2}>0$ being solutions (17)-(22). Applying Itô formula for $V_{1}(x(t), v(t))$ along the trajectory of the following system

$$
\begin{gathered}
d\left[\begin{array}{l}
x(t) \\
v(t)
\end{array}\right]=\left[\begin{array}{cc}
A & G \\
0 & H
\end{array}\right]\left[\begin{array}{l}
x(t) \\
v(t)
\end{array}\right] d t \\
+\left[\begin{array}{cc}
A_{1} & G_{1} \\
0 & H_{1}
\end{array}\right]\left[\begin{array}{l}
x(t) \\
v(t)
\end{array}\right] d w(t), \\
{\left[\begin{array}{c}
x(0) \\
v(0)
\end{array}\right]=\left[\begin{array}{l}
x_{0} \\
v_{0}
\end{array}\right] \in \mathbf{R}^{n+l},}
\end{gathered}
$$

it follows

$$
\begin{aligned}
\mathscr{L} V_{1}(x(t), v(t)) \\
=(A x(t)+G v(t))^{T} \widetilde{Q}_{1}^{-1} x(t) \\
+x^{T}(t) \widetilde{Q}_{1}^{-1}(A x(t)+G v(t)) \\
+\left(A_{1} x(t)+G_{1} v(t)\right)^{T} \widetilde{Q}_{1}^{-1}\left(A_{1} x(t)+G_{1} v(t)\right) \\
+(H v(t))^{T} Q_{2} v(t)+v^{T}(t) Q_{2} H v(t) \\
+\left(H_{1} v(t)\right)^{T} Q_{2} H_{1} v(t),
\end{aligned}
$$


which leads to

$$
\begin{gathered}
\mathscr{L} V_{1}(x(t), v(t))-\alpha V_{1}(x(t), v(t)) \\
=x^{T}(t)\left[A^{T} \widetilde{Q}_{1}^{-1}+\widetilde{Q}_{1}^{-1} A+A_{1}^{T} \widetilde{Q}_{1}^{-1} A_{1}-\alpha \widetilde{Q}_{1}^{-1}\right] x(t) \\
+x^{T}(t)\left[\widetilde{Q}_{1}^{-1} G+A_{1}^{T} \widetilde{Q}_{1}^{-1} G_{1}\right] v(t) \\
+v^{T}(t)\left[G^{T} \widetilde{Q}_{1}^{-1}+G_{1}^{T} \widetilde{Q}_{1}^{-1} A_{1}\right] x(t) \\
+v^{T}(t)\left[G_{1}^{T} \widetilde{Q}_{1}^{-1} G_{1}+H^{T} \widetilde{Q}_{2}+\widetilde{Q}_{2} H\right. \\
\left.+H_{1}^{T} \widetilde{Q}_{2} H_{1}-\alpha \widetilde{Q}_{2}\right] v(t) .
\end{gathered}
$$

Pre- and postmultiplying (17) by $\operatorname{diag}\left\{\widetilde{Q}_{1}^{-1} I \widetilde{Q}_{1}^{-1}\right\}$, according to Schur complement, it is easy to obtain that (17) is equivalent to

$$
\left[\begin{array}{cc}
\Omega+A_{1}^{T} \widetilde{Q}_{1}^{-1} A_{1} & \widetilde{Q}_{1}^{-1} G+A_{1}^{T} \widetilde{Q}_{1}^{-1} G_{1} \\
* & \bar{\Omega}+G_{1}^{T} \widetilde{Q}_{1}^{-1} G_{1}
\end{array}\right]<0,
$$

where $\Omega=A^{T} \widetilde{Q}_{1}^{-1}+\widetilde{Q}_{1}^{-1} A-\alpha \widetilde{Q}_{1}^{-1}, \bar{\Omega}=H^{T} \widetilde{Q}_{2}+\widetilde{Q}_{2} H+$ $H_{1}^{T} \widetilde{Q}_{2} H_{1}-\alpha \widetilde{Q}_{2}$.

By (26) and (27), we obtain

$$
\mathscr{L} V_{1}(x(t), v(t))<\alpha V_{1}(x(t), v(t)) .
$$

Integrating both sides of (28) from 0 to $t$ with $t \in[0, T]$ and then taking mathematical expectation, it yields

$$
\begin{aligned}
\mathbb{E} V_{1}(x(t), v(t))< & \mathbb{E} V_{1}(x(0), v(0)) \\
& +\alpha \int_{0}^{t} \mathbb{E} V_{1}(x(s), v(s)) d s
\end{aligned}
$$

By Lemma 9, we conclude that

$$
\mathbb{E} V_{1}(x(t), v(t))<\mathbb{E} V_{1}(x(0), v(0)) e^{\alpha t} .
$$

By given conditions, it follows

$$
\begin{aligned}
& \mathbb{E} V_{1}(x(0), v(0)) e^{\alpha t} \\
& =\mathbb{E}\left[x^{T}(0) \widetilde{Q}_{1}^{-1} x(0)+v^{T}(0) \widetilde{Q}_{2} v(0)\right] e^{\alpha t} \\
& =\mathbb{E}\left[x^{T}(0) R^{1 / 2} Q_{1}^{-1} R^{1 / 2} x(0)\right. \\
& \left.\quad+v^{T}(0) R_{1}^{1 / 2} Q_{2} R_{1}^{1 / 2} v(0)\right] e^{\alpha t} \\
& \leq \mathbb{E}\left[\lambda_{\max }\left(Q_{1}^{-1}\right) c_{4}+\lambda_{\max }\left(Q_{2}\right) h_{1}\right] e^{\alpha T} \\
& =\left[\frac{1}{\lambda_{\min }\left(Q_{1}\right)} c_{4}+\lambda_{\max }\left(Q_{2}\right) h_{1}\right] e^{\alpha T} \\
& <\left[\frac{1}{\lambda_{1}} c_{4}+\lambda_{2} h_{1}\right] e^{\alpha T},
\end{aligned}
$$

$$
\begin{aligned}
\mathbb{E} V_{1} & (x(t), v(t)) \\
& =\mathbb{E}\left[x^{T}(t) \widetilde{Q}_{1}^{-1} x(t)+v^{T}(t) \widetilde{Q}_{2} v(t)\right] \\
& =\mathbb{E}\left[x^{T}(t) R^{1 / 2} Q_{1}^{-1} R^{1 / 2} x(t)+v^{T}(t) R_{1}^{1 / 2} Q_{2} R_{1}^{1 / 2} v(t)\right] \\
& \geq \mathbb{E}\left[\lambda_{\min }\left(Q_{1}^{-1}\right) x^{T}(t) R x(t)+\lambda_{\min }\left(Q_{2}\right) v^{T}(t) R_{1} v(t)\right] \\
& =\mathbb{E}\left[\frac{1}{\lambda_{\max }\left(Q_{1}\right)} x^{T}(t) R x(t)+\lambda_{\min }\left(Q_{2}\right) v^{T}(t) R_{1} v(t)\right] \\
& >\mathbb{E}\left[x^{T}(t) R x(t)\right] .
\end{aligned}
$$

According to (30) and (31),

$$
\mathbb{E}\left[x^{T}(t) R x(t)\right]<\left(\frac{1}{\lambda_{1}} c_{4}+\lambda_{2} h_{1}\right) e^{\alpha T} .
$$

From (19), we have

$$
\left(\frac{1}{\lambda_{1}} c_{4}+\lambda_{2} h_{1}\right) e^{\alpha T}<c_{2}
$$

From (32) and (33), it is easy to obtain

$$
\mathbb{E}\left[x^{T}(t) R x(t)\right]<c_{2} .
$$

Step 2. $c_{3}<\mathbb{E}\left[x^{T}(0) R x(0)\right] \Rightarrow c_{1}<\mathbb{E}\left[x^{T}(t) R x(t)\right]$.

Take a quadratic function different from $V_{1}(x(t), v(t))$

$$
V_{2}(x(t), v(t))=x^{T}(t) \widetilde{Q}_{1}^{-1} x(t)+v^{T}(t) \widetilde{Q}_{3} v(t),
$$

where $\widetilde{Q}_{1}=R^{-1 / 2} Q_{1} R^{-1 / 2}, \widetilde{Q}_{3}=R_{1}^{1 / 2} Q_{3} R_{1}^{1 / 2}$ with $Q_{1}>0$, $Q_{3}>0$ being solutions (17)-(22). Applying Itô formula for $V_{2}(x(t), v(t))$ along the trajectory of the system of (24), it follows

$$
\begin{aligned}
\mathscr{L} V_{2} & x(t), v(t)) \\
= & (A x(t)+G v(t))^{T} \widetilde{Q}_{1}^{-1} x(t) \\
& +x^{T}(t) \widetilde{Q}_{1}^{-1}(A x(t)+G v(t)) \\
& +\left(A_{1} x(t)+G_{1} v(t)\right)^{T} \widetilde{Q}_{1}^{-1}\left(A_{1} x(t)+G_{1} v(t)\right) \\
& +(H v(t))^{T} Q_{3} v(t)+v^{T}(t) Q_{3} H v(t) \\
& +\left(H_{1} v(t)\right)^{T} Q_{3} H_{1} v(t),
\end{aligned}
$$

which leads to

$$
\begin{gathered}
\beta V_{2}(x(t), v(t))-\mathscr{L} V_{2}(x(t), v(t)) \\
=x^{T}(t)\left[\beta \widetilde{Q}_{1}^{-1}-A^{T} \widetilde{Q}_{1}^{-1}-\widetilde{Q}_{1}^{-1} A-A_{1}^{T} \widetilde{Q}_{1}^{-1} A_{1}\right] x(t) \\
-x^{T}(t)\left[\widetilde{Q}_{1}^{-1} G+A_{1}^{T} \widetilde{Q}_{1}^{-1} G_{1}\right] v(t) \\
-v^{T}(t)\left[G^{T} \widetilde{Q}_{1}^{-1}+G_{1}^{T} \widetilde{Q}_{1}^{-1} A_{1}\right] x(t) \\
+v^{T}(t)\left[\beta \widetilde{Q}_{3}-G_{1}^{T} \widetilde{Q}_{1}^{-1} G_{1}-H^{T} \widetilde{Q}_{3}\right. \\
\left.-\widetilde{Q}_{3} H-H_{1}^{T} \widetilde{Q}_{3} H_{1}\right] v(t) .
\end{gathered}
$$


Pre- and postmultiplying (18) by $\operatorname{diag}\left\{\widetilde{Q}_{1}^{-1} I \widetilde{Q}_{1}^{-1}\right\}$, by Schur complement, it is easily obtained that (18) is equivalent to

$$
\left[\begin{array}{cc}
\bar{\Sigma}+A_{1}^{T} \widetilde{Q}_{1}^{-1} A_{1} & \widetilde{Q}_{1}^{-1} G+A_{1}^{T} \widetilde{Q}_{1}^{-1} G_{1} \\
* & \bar{\Sigma}+G_{1}^{T} \widetilde{Q}_{1}^{-1} G_{1}
\end{array}\right]<0,
$$

where $\bar{\Sigma}=\beta \widetilde{Q}_{1}^{-1}-A^{T} \widetilde{Q}_{1}^{-1}-\widetilde{Q}_{1}^{-1} A, \bar{\Sigma}=\beta \widetilde{Q}_{3}-H^{T} \widetilde{Q}_{3}-\widetilde{Q}_{3} H-$ $H_{1}^{T} \widetilde{Q}_{3} H_{1}$.

Considering (37) and (38), we obtain

$$
\mathscr{L} V_{2}(x(t), v(t))>\beta V_{2}(x(t), v(t)) \text {. }
$$

Integrating both sides of (39) from 0 to $t$ with $t \in[0, T]$ and then taking the mathematical expectation, it yields

$$
\begin{aligned}
\mathbb{E} V_{2}(x(t), v(t))> & \mathbb{E} V_{2}(x(0), v(0)) \\
& +\beta \int_{0}^{t} \mathbb{E} V_{2}(x(s), v(s)) d s .
\end{aligned}
$$

By Lemma 9, we conclude that

$$
\mathbb{E} V_{2}(x(t), v(t))>\mathbb{E} V_{2}(x(0), v(0)) e^{\beta t} .
$$

By given conditions, it follows

$$
\begin{aligned}
& \mathbb{E} V_{2}(x(0), v(0)) e^{\beta t} \\
&= {\left[x^{T}(0) \widetilde{Q}_{1}^{-1} x(0)+v^{T}(0) \widetilde{Q}_{3} v(0)\right] e^{\beta t} } \\
&= {\left[x^{T}(0) R^{1 / 2} Q_{1}^{-1} R^{1 / 2} x(0)\right.} \\
&\left.\quad+v^{T}(0) R_{1}^{1 / 2} Q_{3} R_{1}^{1 / 2} v(0)\right] e^{\beta t} \\
& \geq \lambda_{\min }\left(Q_{1}^{-1}\right) c_{3}=\frac{1}{\lambda_{\max }\left(Q_{1}\right)} c_{3}>c_{3}, \\
& \mathbb{E} V_{2}(x(t), v(t)) \\
&=\mathbb{E}\left[x^{T}(t) \widetilde{Q}_{1}^{-1} x(t)+v^{T}(t) \widetilde{Q}_{3} v(t)\right] \\
&=\mathbb{E}\left[x^{T}(t) R^{1 / 2} Q_{1}^{-1} R^{1 / 2} x(t)+v^{T}(t) R_{1}^{1 / 2} Q_{3} R_{1}^{1 / 2} v(t)\right] \\
& \leq \mathbb{E}\left[\lambda_{\max }\left(Q_{1}^{-1}\right) x^{T}(t) R x(t)+\lambda_{\max }\left(Q_{3}\right) v^{T}(t) R_{1} v(t)\right] \\
&=\mathbb{E}\left[\frac{1}{\lambda_{\min }\left(Q_{1}\right)} x^{T}(t) R x(t)+\lambda_{\max }\left(Q_{3}\right) v^{T}(t) R_{1} v(t)\right] \\
&<\frac{1}{\lambda_{1}} \mathbb{E}\left[x^{T}(t) R x(t)\right]+\lambda_{3} h .
\end{aligned}
$$

By (41) and (42),

$$
c_{3}<\frac{1}{\lambda_{1}} \mathbb{E}\left[x^{T}(t) R x(t)\right]+\lambda_{3} h .
$$

From (20), we have

$$
c_{1}-c_{3} \lambda_{1}+h \lambda_{3} \lambda_{1}<0
$$

that is,

$$
c_{1}<\left[c_{3}-\lambda_{3} h\right] \lambda_{1}
$$

From (43) and (45), it is easy to obtain

$$
c_{1}<\mathbb{E}\left[x^{T}(t) R x(t)\right] .
$$

Therefore, the proof of the theorem is completed.

Remark 11. Theorem 10 can be employed for testing finitetime stochastic boundedness of system (1).

\section{Finite-Time Stochastic Bounded Controller Design}

In this section, we use different quadratic function approach to design state and output feedback finite-time bounded controller such that the closed-loop system of system (1) is finite-time stochastically bounded over a finite-time interval $[0, T]$, respectively.

4.1. State Feedback Finite-Time Bounded Controller Design. For system (1), we first consider a state feedback controller

$$
u(t)=K x(t)
$$

then the closed-loop system of (1) is as follows:

$$
\begin{aligned}
d x(t)= & {[(A+B K) x(t)+G v(t)] d t } \\
& +\left[\left(A_{1}+B_{1} K\right) x(t)+G_{1} v(t)\right] d w(t)
\end{aligned}
$$

Next, a sufficient condition of the existence for state feedback finite-time bounded controller is presented by Theorem 10 .

Theorem 12. If there exist $\alpha \geq 0, \beta \geq 0$, positive definite matrices $Q_{1} \in \mathbf{R}^{n \times n}, Q_{2} \in \mathbf{R}^{l \times l}, Q_{3} \in \mathbf{R}^{l \times l}$, and a matrix $M \in \mathbf{R}^{m \times n}$, and some scalars $\lambda_{i}>0, i=1,2,3$ such that (19)-(22) and the following inequalities hold

$$
\begin{aligned}
& {\left[\begin{array}{ccc}
\Lambda_{1} & G & \widetilde{Q}_{1} A_{1}^{T}+M^{T} B_{1}^{T} \\
* & \Gamma-\alpha \widetilde{Q}_{2} & G_{1}^{T} \\
* & * & -\widetilde{Q}_{1}
\end{array}\right]<0,} \\
& {\left[\begin{array}{ccc}
\Lambda_{2} & G & \widetilde{Q}_{1} A_{1}^{T}+M^{T} B_{1}^{T} \\
* & \beta \widetilde{Q}_{3}-\bar{\Gamma} & G_{1}^{T} \\
* & * & -\widetilde{Q}_{1}
\end{array}\right]<0,}
\end{aligned}
$$

then system (48) is finite-time stochastically bounded with respect to $\left(c_{1}, c_{2}, c_{3}, c_{4}, \mathscr{W}, T, R\right)$, where $\widetilde{Q}_{1}=R^{-1 / 2} Q_{1} R^{-1 / 2}$, $\widetilde{Q}_{2}=R_{1}^{1 / 2} Q_{2} R_{1}^{1 / 2}, \widetilde{Q}_{3}=R_{1}^{1 / 2} Q_{3} R_{1}^{1 / 2}, \Lambda_{1}=\widetilde{Q}_{1} A^{T}+A \widetilde{Q}_{1}+$ $M^{T} B^{T}+B M-\alpha \widetilde{Q}_{1}, \Lambda_{2}=\beta \widetilde{Q}_{1}-\widetilde{Q}_{1} A^{T}-A \widetilde{Q}_{1}-M^{T} B^{T}-B M$. In this case, a desired controller gain is given by $K=M \widetilde{Q}_{1}^{-1}$. 
Proof. We can replace $A$ by $A+B K$ and $A_{1}$ by $A_{1}+B_{1} K$ in Theorem 10. As a result, conditions (17) and (18)

$$
\begin{aligned}
& {\left[\begin{array}{ccc}
\Lambda_{1}^{*} & G & \widetilde{Q}_{1}\left(A_{1}+B_{1} K\right)^{T} \\
* & \Gamma-\alpha \widetilde{Q}_{2} & G_{1}^{T} \\
* & * & -\widetilde{Q}_{1}
\end{array}\right]<0,} \\
& {\left[\begin{array}{ccc}
\Lambda_{2}^{*} & G & \widetilde{Q}_{1}\left(A_{1}+B_{1} K\right)^{T} \\
* & \beta \widetilde{Q}_{3}-\bar{\Gamma} & G_{1}^{T} \\
* & * & -\widetilde{Q}_{1}
\end{array}\right]<0}
\end{aligned}
$$

hold, where $\Lambda_{1}^{*}=-\alpha \widetilde{Q}_{1}+\widetilde{Q}_{1}(A+B K)^{T}+(A+B K) \widetilde{Q}_{1}, \Lambda_{2}^{*}=$ $\beta \widetilde{Q}_{1}-\widetilde{Q}_{1}(A+B K)^{T}-(A+B K) \widetilde{Q}_{1}$. Letting $M=K \widetilde{Q}_{1}$, it can be seen that (49) and (50) are derived from (51) and (52), respectively. This completes the proof.

4.2. Dynamic Output Feedback Finite-Time Bounded Controller Design. When the system states are not completely accessible, state feedback controllers may become invalid. This motivates us to propose an output-feedback controller. Without loss of generality, we can assume the following.

Assumption 13. There exists a state feedback controller $u(t)=K x(t)$ which has been designed using the results of Theorem 12.

We choose, as usual, a finite-dimensional observer-based controller as follows:

$$
\begin{gathered}
d \widehat{x}(t)=[A \widehat{x}(t)+B u(t)+L(y(t)-C \hat{x}(t))] d t \\
+\left[A_{1} \widehat{x}(t)+B_{1} u(t)+L(y(t)-C \widehat{x}(t))\right] d w(t), \\
u(t)=K \widehat{x}(t), \quad \widehat{x}(0)=\widehat{x}_{0} \in \mathbf{R}^{n},
\end{gathered}
$$

where $\hat{x}(t) \in \mathbf{R}^{n}$ is the the estimate of the state of $x(t)$ and $L$ is the estimator gain matrix with appropriate dimensions, which is to be determined.

$$
\begin{aligned}
& \text { Let } e(t)=x(t)-\widehat{x}(t) ; \text { then we obtain the error system } \\
& \qquad \begin{aligned}
d e(t)= & {[(A-L C) e(t)+G v(t)] d t } \\
& +\left[\left(A_{1}-L C\right) e(t)+G_{1} v(t)\right] d w(t)
\end{aligned}
\end{aligned}
$$

In practice, we hope the error $e(t)$ is as small as possible. As usual, it is required to satisfy $\mathbb{E}\left[e^{T}(t) \operatorname{Re}(t)\right]<1, t \in[0, T]$.

Let $z(t)=\left[x^{T}(t) e^{T}(t) v^{T}(t)\right]^{T}$; then the resulting argument system becomes

$$
\begin{gathered}
d z(t)=\bar{A} z(t) d t+\bar{A}_{1} z(t) d w(t), \\
z(0)=\left[\begin{array}{lll}
x_{0}^{T} & e_{0}^{T} & v_{0}^{T}
\end{array}\right]^{T} \in \mathbf{R}^{2 n+l}
\end{gathered}
$$

where

$$
\begin{gathered}
\bar{A}=\left[\begin{array}{ccc}
A+B K & -B K & G \\
0 & A-L C & G \\
0 & 0 & H
\end{array}\right], \\
\bar{A}_{1}=\left[\begin{array}{ccc}
A_{1}+B_{1} K & -B_{1} K & G_{1} \\
0 & A_{1}-L C & G_{1} \\
0 & 0 & H_{1}
\end{array}\right] .
\end{gathered}
$$

Now, on the basis of Assumption 13, the following theorem gives a sufficient condition of the existence of $L$.

Theorem 14. If there exist $\alpha \geq 0, \beta \geq 0$, positive matrices $Q_{k}(k=1, \ldots, 5)$, a matrix $N$, and positive scalars $\lambda_{i}(i=$ $1, \ldots, 7)$, such that the following inequalities hold

$$
\begin{aligned}
& {\left[\begin{array}{ccc}
\Pi_{11}-\alpha \widetilde{Q}_{1} & \Pi_{12} & \Pi_{13} \\
* & \Pi_{22}-\alpha \widetilde{Q}_{2} & \Pi_{23}+A^{T} \widetilde{Q}_{2}-C^{T} N^{T} \\
* & * & \Pi_{33}-\widetilde{Q}_{2}-\alpha \widetilde{Q}_{3}
\end{array}\right]<0} \\
& {\left[\begin{array}{ccc}
\beta \widetilde{Q}_{4}-\digamma_{11} & -\digamma_{12} & \digamma_{13} \\
* & \beta \widetilde{Q}_{2}-\digamma_{22} & -\digamma_{23} \\
* & * & \beta \widetilde{Q}_{5}-\digamma_{33}
\end{array}\right]<0,} \\
& \lambda_{4} I<Q_{1}<\lambda_{1} I, \quad 0<Q_{2}<\lambda_{2} I, \quad 0<Q_{3}<\lambda_{3} I, \\
& \lambda_{6} I<Q_{4}<\lambda_{5} I \\
& 0<Q_{5}<\lambda_{7} I, \\
& \lambda_{1} c_{4}+\lambda_{2}+\lambda_{3} h_{1} \leq c_{2} \exp (-\alpha T) \lambda_{4}, \\
& c_{1} \lambda_{5}-c_{3} \lambda_{6}+\lambda_{2}+\lambda_{7} h<0
\end{aligned}
$$

then the closed-loop system (55) is finite-time stochastically bounded with respect to $\left(c_{1}, c_{2}, c_{3}, c_{4}, \mathscr{W}, T, R\right)$. In this case, $L=\widetilde{Q}_{2}^{-1} N$, where

$$
\begin{aligned}
& \widetilde{Q}_{1}=R^{1 / 2} Q_{1} R^{1 / 2}, \quad \widetilde{Q}_{2}=R^{1 / 2} Q_{2} R^{1 / 2}, \quad \widetilde{Q}_{3}=R_{1}^{1 / 2} Q_{3} R_{1}^{1 / 2} \\
& \widetilde{Q}_{4}=R^{1 / 2} Q_{4} R^{1 / 2}, \quad \widetilde{Q}_{5}=R_{1}^{1 / 2} Q_{5} R_{1}^{1 / 2}, \\
& \Pi_{11}=(A+B K)^{T} \widetilde{Q}_{1}+\widetilde{Q}_{1}(A+B K) \\
& +\left(A_{1}+B_{1} K\right)^{T} \widetilde{Q}_{1}\left(A_{1}+B_{1} K_{1}\right), \\
& \Pi_{12}=\left(A_{1}+B_{1} K\right)^{T} \widetilde{Q}_{1} B_{1} K+\widetilde{Q}_{1} B K, \\
& \Pi_{13}=\widetilde{Q}_{1} G+\left(A_{1}+B_{1} K\right)^{T} \widetilde{Q}_{1} G_{1} \text {, } \\
& \Pi_{22}=K^{T} B_{1}^{T} \widetilde{Q}_{1} B_{1} K+A^{T} \widetilde{Q}_{2}+\widetilde{Q}_{2} A-C^{T} N-N^{T} C, \\
& \Pi_{23}=-K^{T} B_{1}^{T} \widetilde{Q}_{1} G_{1}+\widetilde{Q}_{2} G_{1}+A^{T} \widetilde{Q}_{2} G_{1}-C^{T} N^{T} G_{1} \text {, } \\
& \Pi_{33}=H^{T} \widetilde{Q}_{3}+\widetilde{Q}_{3} H+H_{1}^{T} \widetilde{Q}_{3} H_{1}+G_{1}^{T} \widetilde{Q}_{1} G_{1}, \\
& F_{11}=(A+B K)^{T} \widetilde{Q}_{4}+\widetilde{Q}_{4}(A+B K) \\
& +\left(A_{1}+B_{1} K\right)^{T} \widetilde{Q}_{4}\left(A_{1}+B_{1} K_{1}\right), \\
& F_{12}=\left(A_{1}+B_{1} K\right)^{T} \widetilde{Q}_{4} B_{1} K+\widetilde{Q}_{4} B K, \\
& F_{13}=\widetilde{Q}_{4} G+\left(A_{1}+B_{1} K\right)^{T} \widetilde{Q}_{4} G_{1}, \\
& F_{22}=K^{T} B_{1}^{T} \widetilde{Q}_{4} B_{1} K+A^{T} \widetilde{Q}_{2}+\widetilde{Q}_{2} A-C^{T} N-N^{T} C, \\
& F_{23}=K^{T} B_{1}^{T} \widetilde{Q}_{4} G_{1}+\widetilde{Q}_{2} G_{1}+A^{T} \widetilde{Q}_{2} G_{1}-C^{T} N^{T} G_{1}, \\
& F_{33}=H^{T} \widetilde{Q}_{5}+\widetilde{Q}_{5} H+H_{1}^{T} \widetilde{Q}_{5} H_{1}+G_{1}^{T} \widetilde{Q}_{4} G_{1} .
\end{aligned}
$$


Proof.

Step 1. $\mathbb{E}\left[x^{T}(0) R x(0)\right]<c_{4} \Rightarrow \mathbb{E}\left[x(t)^{T} R x(t)\right]<c_{2}$.

Let $P=\operatorname{diag}\left\{\widetilde{Q}_{1}, \widetilde{Q}_{2}, \widetilde{Q}_{3}\right\}$ with $Q_{1}>0, Q_{2}>0, Q_{3}>0$ being solutions to (57)-(63), and $z(t)=\left[x^{T}(t) e^{T}(t) v^{T}(t)\right]^{T}$; we have

$$
\begin{aligned}
V_{3}(z(t))= & z^{T}(t) P z(t) \\
= & x^{T}(t) \widetilde{Q}_{1} x(t)+e^{T}(t) \widetilde{Q}_{2} e(t)+v^{T}(t) \widetilde{Q}_{3} v(t) \\
= & x^{T}(t) R^{1 / 2} Q_{1} R^{1 / 2} x(t)+e^{T}(t) R^{1 / 2} Q_{2} R^{1 / 2} e(t) \\
& +v^{T}(t) R_{1}^{1 / 2} Q_{3} R_{1}^{1 / 2} v(t) .
\end{aligned}
$$

Applying Itô formula for $V_{3}(z(t))$ along with the state trajectory of (55), we obtain

$$
\begin{aligned}
\mathscr{L} V_{3} & (z(t)) \\
= & {[(A+B K) x(t)-B K e(t)+G v(t)]^{T} \widetilde{Q}_{1} x(t) } \\
& +x^{T}(t) \widetilde{Q}_{1}[(A+B K) x(t)-B K e(t)+G v(t)] \\
& +\left[\left(A_{1}+B_{1} K\right) x(t)-B_{1} K e(t)+G_{1} v(t)\right]^{T} \widetilde{Q}_{1} \\
& \times\left[\left(A_{1}+B_{1} K\right) x(t)-B_{1} K e(t)+G_{1} v(t)\right] \\
& +\left[(A-L C) e(t)+G_{1} v(t)\right]^{T} \widetilde{Q}_{2} e(t) \\
& +e^{T}(t) \widetilde{Q}_{2}\left[(A-L C) e(t)+G_{1} v(t)\right] \\
& +\left[\left(A_{1}-L C\right) e(t)+G_{1} v(t)\right]^{T} \widetilde{Q}_{2} \\
& \times\left[\left(A_{1}-L C\right) e(t)+G_{1} v(t)\right] \\
& +v^{T}(t) H^{T} \widetilde{Q}_{3} v(t)+v^{T}(t) \widetilde{Q}_{3} H v(t) \\
& +v^{T}(t) H_{1}^{T} \widetilde{Q}_{3} H_{1} v(t) \\
= & {\left[x^{T}(t) e^{T}(t) v^{T}(t)\right]^{T} Z[x(t) e(t) v(t)], }
\end{aligned}
$$

where

$$
\begin{gathered}
Z=\left[\begin{array}{ccc}
\Pi_{11} & \Pi_{12} & \Pi_{13} \\
* & \Xi_{22} & \Xi_{23} \\
* & * & \Xi_{33}
\end{array}\right], \\
\Xi_{22}=K^{T} B_{1}^{T} \widetilde{Q}_{1} B_{1} K+A^{T} \widetilde{Q}_{2}+\widetilde{Q}_{2} A-C^{T} L^{T} \widetilde{Q}_{2}-\widetilde{Q}_{2} L C, \\
\Xi_{23}=-K^{T} B_{1}^{T} \widetilde{Q}_{1} G_{1}+\widetilde{Q}_{2} G_{1}+A^{T} \widetilde{Q}_{2} G_{1}-C^{T} L^{T} \widetilde{Q}_{2} G_{1} \\
+(A-L C)^{T} \widetilde{Q}_{2}(A-L C), \\
\Xi_{33}=H^{T} \widetilde{Q}_{3}+\widetilde{Q}_{3} H+H_{1}^{T} \widetilde{Q}_{3} H_{1}+G_{1}^{T} \widetilde{Q}_{1} G_{1} .
\end{gathered}
$$

According to Schur complement, by letting $N=\widetilde{Q}_{2} L$, condition (57) can be rewritten as

$$
\left[\begin{array}{ccc}
\Pi_{11} & \Pi_{12} & \Pi_{13} \\
* & \Xi_{22} & \Xi_{23} \\
* & * & \Xi_{33}
\end{array}\right]<\left[\begin{array}{ccc}
\alpha \widetilde{Q}_{1} & 0 & 0 \\
0 & \alpha \widetilde{Q}_{2} & 0 \\
0 & 0 & \alpha \widetilde{Q}_{3}
\end{array}\right] .
$$

It is obvious that (68) gives

$$
\mathscr{L} V_{3}(z(t))<\alpha V_{3}(z(t)), \quad t \in[0, T] .
$$

By integrating inequality (69) between 0 and $t$, and taking the mathematical expectation, it follows that

$$
\mathbb{E} V_{3}(z(t))<\mathbb{E} V_{3}(z(0))+\alpha \int_{0}^{t} \mathbb{E} V_{3}(z(s)) d s .
$$

By Lemma 8, we have

$$
\mathbb{E} V_{3}(z(t))<\mathbb{E} V_{3}(z(0)) e^{\alpha t} .
$$

Considering (59), we obtain

$$
\begin{aligned}
& \mathbb{E} V_{3}(z(t)) \\
&= \mathbb{E}\left[x^{T}(t) R^{1 / 2} Q_{1} R^{1 / 2} x(t)+e^{T}(t) R^{1 / 2} Q_{2} R^{1 / 2} e(t)\right. \\
&\left.\quad+v^{T}(t) R_{1}^{1 / 2} Q_{3} R_{1}^{1 / 2} v(t)\right] \\
& \geq \mathbb{E}\left[x^{T}(t) R^{1 / 2} Q_{1} R^{1 / 2} x(t)\right] \\
& \geq \lambda_{\min }\left(Q_{1}\right) \mathbb{E}\left[x^{T}(t) R x(t)\right] \\
&> \lambda_{4} \mathbb{E}\left[x^{T}(t) R x(t)\right], \\
& V_{3}(z(0)) e^{\alpha t}\left(x^{T}(0) R^{1 / 2} Q_{1} R^{1 / 2} x(0)+e^{T}(0) R^{1 / 2} Q_{2} R^{1 / 2} e(0)\right. \\
&\left.\quad+v^{T}(0) R_{1}^{1 / 2} Q_{3} R_{1}^{1 / 2} v(0)\right) e^{\alpha t} \\
& \leq\left(\lambda_{\max }\left(Q_{1}\right) x^{T}(0) R x(0)+\lambda_{\max }\left(Q_{2}\right) e^{T}(0) R e(0)\right. \\
&\left.\quad+\lambda_{\max }\left(Q_{3}\right) v^{T}(0) R_{1} v(0)\right) e^{\alpha T} \\
& \leq\left(\lambda_{\max }\left(Q_{1}\right) c_{4}+\lambda_{\max }\left(Q_{2}\right)+\lambda_{\max }\left(Q_{3}\right) h_{2}\right) e^{\alpha T} \\
&<\left(\lambda_{1} c_{4}+\lambda_{2}+\lambda_{3} h_{1}\right) e^{\alpha T} .
\end{aligned}
$$

According to (72), it follows

$$
\mathbb{E}\left[x^{T}(t) R x(t)\right]<\frac{1}{\lambda_{4}}\left(\lambda_{1} c_{4}+\lambda_{2}+\lambda_{3} h_{2}\right) e^{\alpha T} .
$$

By (73) and condition (62), it follows $\mathbb{E}\left[x^{T}(t) R x(t)\right]<\mathcal{c}_{2}$ for all $t \in[0, T]$.

Step 2. $c_{3}<\mathbb{E}\left[x^{T}(0) R x(0)\right] \Rightarrow c_{1}<\mathbb{E}\left[x^{T}(t) R x(t)\right]$. 
Let $\bar{P}=\operatorname{diag}\left\{\widetilde{Q}_{4} \widetilde{Q}_{2} \widetilde{Q}_{5}\right\}$ with $Q_{4}>0, Q_{2}>0, Q_{5}>0$ being solutions to (57)-(63), and $z(t)=\left[x^{T}(t) e^{T}(t) v^{T}(t)\right]^{T}$; we have

$$
\begin{aligned}
V_{4}(z(t))= & z^{T}(t) P z(t) \\
= & x^{T}(t) \widetilde{Q}_{4} x(t)+e^{T}(t) \widetilde{Q}_{2} e(t)+v^{T}(t) \widetilde{Q}_{5} v(t) \\
= & x^{T}(t) R^{1 / 2} Q_{4} R^{1 / 2} x(t)+e^{T}(t) R^{1 / 2} Q_{2} R^{1 / 2} e(t) \\
& +v^{T}(t) R_{1}^{1 / 2} Q_{5} R_{1}^{1 / 2} v(t) .
\end{aligned}
$$

Applying Itô formula for $V_{4}(z(t))$ along with the state trajectory of (55), we obtain

$$
\begin{aligned}
\mathscr{L} V_{4} & (z(t)) \\
= & {[(A+B K) x(t)-B K e(t)+G v(t)]^{T} \widetilde{Q}_{4} x(t) } \\
& +x^{T}(t) \widetilde{Q}_{4}[(A+B K) x(t)-B K e(t)+G v(t)] \\
& +\left[\left(A_{1}+B_{1} K\right) x(t)-B_{1} K e(t)+G_{1} v(t)\right]^{T} \widetilde{Q}_{4} \\
& \times\left[\left(A_{1}+B_{1} K\right) x(t)-B_{1} K e(t)+G_{1} v(t)\right] \\
& +\left[(A-L C) e(t)+G_{1} v(t)\right]^{T} \widetilde{Q}_{2} e(t) \\
& +e^{T}(t) \widetilde{Q}_{2}\left[(A-L C) e(t)+G_{1} v(t)\right] \\
& +\left[\left(A_{1}-L C\right) e(t)+G_{1} v(t)\right]^{T} \widetilde{Q}_{2} \\
& \times\left[\left(A_{1}-L C\right) e(t)+G_{1} v(t)\right]+v^{T}(t) H^{T} \widetilde{Q}_{5} v(t) \\
& +v^{T}(t) \widetilde{Q}_{5} H v(t)+v^{T}(t) H_{1}^{T} \widetilde{Q}_{5} H_{1} v(t) \\
= & {\left[x^{T}(t) e^{T}(t) v^{T}(t)\right]^{T} \bar{Z}[x(t) e(t) v(t)], }
\end{aligned}
$$

where

$$
\begin{gathered}
\bar{Z}=\left[\begin{array}{ccc}
F_{11} & F_{12} & F_{13} \\
* & \bar{F}_{22} & \bar{F}_{23} \\
* & * & \bar{F}_{33}
\end{array}\right], \\
\bar{F}_{22}=K^{T} B_{1}^{T} \widetilde{Q}_{4} B_{1} K+A^{T} \widetilde{Q}_{2}+\widetilde{Q}_{2} A-C^{T} L^{T} \widetilde{Q}_{2}-\widetilde{Q}_{2} L C, \\
\bar{F}_{23}=-K^{T} B_{1}^{T} \widetilde{Q}_{4} G_{1}+\widetilde{Q}_{2} G_{1}+A^{T} \widetilde{Q}_{2} G_{1}-C^{T} L^{T} \widetilde{Q}_{2} G_{1} \\
+(A-L C)^{T} \widetilde{Q}_{2}(A-L C), \\
\bar{F}_{33}=H^{T} \widetilde{Q}_{5}+\widetilde{Q}_{5} H+H_{1}^{T} \widetilde{Q}_{5} H_{1}+G_{1}^{T} \widetilde{Q}_{4} G_{1} .
\end{gathered}
$$

Note that (58) implies

$$
\beta V_{4}(z(t))<\mathscr{L} V_{4}(z(t)) .
$$

Integrating both sides of (77) from 0 to $t$ with $t \in[0, T]$ and then taking the mathematical expectation, it yields

$$
\mathbb{E} V_{4}(z(0))+\beta \int_{0}^{t} \mathbb{E} V_{4}(z(s)) d s<\mathbb{E} V_{4}(z(t)) .
$$

By Lemma 9,

$$
\mathbb{E} V_{4}(z(0)) e^{\beta t}<\mathbb{E} V_{4}(z(t)) .
$$

Considering (59), we obtain

$$
\begin{aligned}
& \mathbb{E} V_{4}(z(t)) \\
&= \mathbb{E}\left[x^{T}(t) R^{1 / 2} Q_{4} R^{1 / 2} x(t)+e^{T}(t) R^{1 / 2} Q_{2} R^{1 / 2} e(t)\right. \\
&\left.\quad v^{T}(t) R_{1}^{1 / 2} Q_{5} R_{1}^{1 / 2} v(t)\right] \\
& \leq \lambda_{\max }\left(Q_{4}\right) \mathbb{E}\left[x^{T}(t) R x(t)\right]+\lambda_{\max }\left(Q_{2}\right) \mathbb{E}\left[e^{T}(t) R e(t)\right] \\
&+\lambda_{\max }\left(Q_{5}\right) \mathbb{E}\left[v^{T}(t) R v(t)\right] \\
&< \lambda_{5} \mathbb{E}\left[x^{T}(t) R x(t)\right]+\lambda_{2}+\lambda_{7} h, \\
& V_{4}(z(0)) e^{\alpha t} \\
&=\left(x^{T}(0) R^{1 / 2} Q_{4} R^{1 / 2} x(0)+e^{T}(0) R^{1 / 2} Q_{2} R^{1 / 2} e(0)\right. \\
&\left.\quad+v^{T}(0) R_{1}^{1 / 2} Q_{5} R_{1}^{1 / 2} v(0)\right) e^{\alpha t} \\
& \geq \lambda_{\min }\left(Q_{4}\right) x^{T}(0) R x(0)+\lambda_{\min }\left(Q_{2}\right) e^{T}(0) R e(0) \\
& \quad+\lambda_{\min }\left(Q_{5}\right) v^{T}(0) R_{1} v(0)>\lambda_{6} \mathcal{c}_{3} .
\end{aligned}
$$

From (79) and (80), we have

$$
\frac{c_{3} \lambda_{6}-\lambda_{2}-\lambda_{7} h}{\lambda_{5}}<\mathbb{E}\left[x^{T}(t) R x(t)\right] .
$$

Equation (63) gives

$$
c_{1}<\frac{c_{3} \lambda_{6}-\lambda_{2}-\lambda_{7} h}{\lambda_{5}}
$$

So we easily obtain

$$
c_{1}<\mathbb{E}\left[x^{T}(t) R x(t)\right]
$$

for all $t \in[0, T]$. This ends the proof.

Remark 15. It is easy to see that the values of $\alpha$ and $\beta$ determine the feasibility of the above Theorems. The procedure of how to choose $\alpha$ and $\beta$ is given in the next section.

\section{Numerical Algorithm}

This section gives an algorithm for the results of the paper. The following algorithm is used to solve the matrix inequalities in Theorem 10. Similar algorithms can be used in Theorem 12 and Theorem 14.

Algorithm 16.

Step 1. Given $c_{1}, c_{2}, c_{3}, c_{4}, R, T, \mathscr{W}$. 


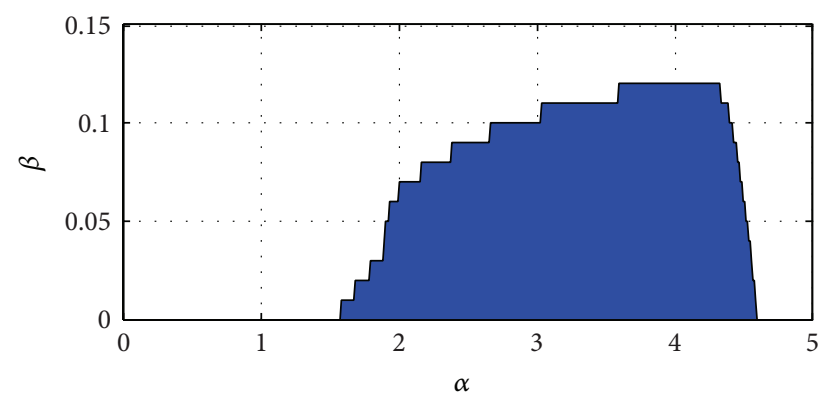

Figure 1: A region by $\alpha$ and $\beta$.

Step 2. Take a series of $\alpha_{i}(i=1, \ldots, n)$ by a step size $d_{1}$ and a series of $\beta_{j}(j=1, \ldots, m)$ by a step size $d_{2}$.

Step 3. Set $i=1$; take a $\alpha_{i}$.

Step 4. Set $j=1$; take a $\beta_{j}$.

Step 5. If $\left(\alpha_{i}, \beta_{j}\right)$ makes (17)-(22) have feasible solutions, then store $\left(\alpha_{i}, \beta_{j}\right)$ into $(X(i), Y(j))$ and $\beta_{j}=\beta_{j+1}$; go to Step 5; otherwise go to Step 6.

Step 6. If $i+1<n$, then $\alpha_{i}=\alpha_{i+1}$ and takes $\beta_{1}$; go to Step 5 . Otherwise, go to Step 7.

Step 7. Stop. If $(X, Y)=(0,0)$, then we cannot find $(\alpha, \beta)$ making (17)-(22) have feasible solution; otherwise, there exists $(\alpha, \beta)$ making (17)-(22) have feasible solution.

Remark 17. By Algorithm 16, we can obtain a region surrounded by $\alpha$ and $\beta$, if it exists, which is used to select $\alpha$ and $\beta$ for appropriate conditions.

\section{Examples}

In this section, an example is given to illustrate the results we have obtained.

Example 1. Applying Algorithm 16 to Theorem 12, we obtain a region surrounded by $\alpha$ and $\beta$, which is illustrated by Figure 1.

Let us consider system (1) with

$$
\begin{gathered}
A=\left[\begin{array}{ll}
1.21 & -2.27 \\
3.57 & -0.82
\end{array}\right], \quad B=\left[\begin{array}{cc}
2 & 1.2 \\
1.5 & -0.1
\end{array}\right], \\
G=\left[\begin{array}{cc}
1.1 & 0.05 \\
0.06 & 0.2
\end{array}\right], \quad A_{1}=\left[\begin{array}{ll}
0.16 & 0.45 \\
0.12 & 0.17
\end{array}\right], \\
B_{1}=\left[\begin{array}{cc}
0.9 & 1 \\
-1 & 1.5
\end{array}\right], \quad G_{1}=\left[\begin{array}{cc}
-0.01 & 0.03 \\
0.02 & -0.12
\end{array}\right], \\
H=\left[\begin{array}{cc}
0.5 & -0.3 \\
-0.6 & 0.5
\end{array}\right], \quad H_{1}=\left[\begin{array}{cc}
0.13 & 0.5 \\
0.15 & 0.18
\end{array}\right], \\
C=\left[\begin{array}{ll}
1 & 2
\end{array}\right], \quad x(0)=\left[\begin{array}{ll}
1.5 & -1.5
\end{array}\right]^{T} .
\end{gathered}
$$

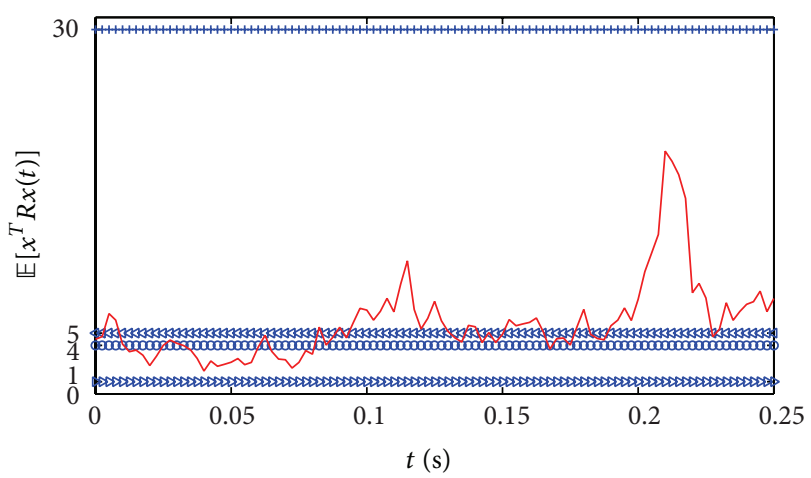

FIGURE 2: The evolution of $\mathbb{E}\left[x^{T}(t) R x(t)\right]$ of the closed-loop system of (1) in Case 1.

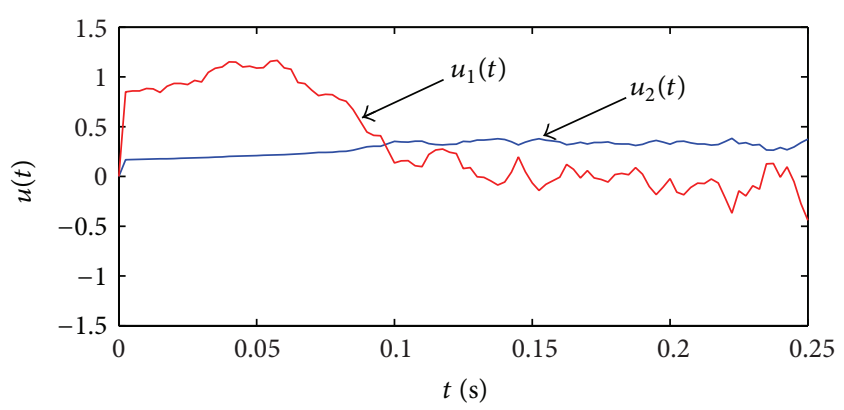

FIgURE 3: The evolution of $u(t)$.

The parameters are given as $c_{1}=1, c_{2}=30, c_{3}=4, c_{4}=5$, $h=0.5, h_{1}=0.1, T=0.25$, and $R=R_{1}=I$.

Case 1 (state feedback finite-time bounded controller design). By Figure 1, selecting $\alpha=3$ and $\beta=0.05$, and solving (19)(22) and (49)-(50), we obtain

$$
\begin{gathered}
\lambda_{1}=0.4967, \quad \lambda_{2}=0.8062, \quad \lambda_{3}=3.8605 \\
Q_{1}=\left[\begin{array}{ll}
0.6544 & 0.2262 \\
0.2262 & 0.8412
\end{array}\right], \quad Q_{2}=\left[\begin{array}{cc}
0.7743 & -0.0438 \\
-0.0438 & 0.3914
\end{array}\right], \\
Q_{3}=\left[\begin{array}{ll}
3.6867 & 0.3789 \\
0.3789 & 1.8994
\end{array}\right], \quad M=\left[\begin{array}{cc}
0.0764 & 0.0293 \\
-0.0007 & -0.3221
\end{array}\right] .
\end{gathered}
$$

Hence, the feedback gain matrix is given by

$$
K=M \widetilde{Q}_{1}^{-1}=\left[\begin{array}{cc}
0.1155 & 0.0038 \\
0.1447 & -0.4218
\end{array}\right] \text {. }
$$

The evolution of $\mathbb{E}\left[x^{T}(t) R x(t)\right]$ of the closed-loop system of (1) is illustrated by Figure 2, which shows that the closedloop system of (1) is finite-time stochastically bounded with respect to $(1,30,4,5,0.25, I, \mathscr{W})$. The corresponding control curves are illustrated by Figure 3.

Case 2 (dynamic output feedback finite-time bounded controller design). Based on state feedback controller design, 


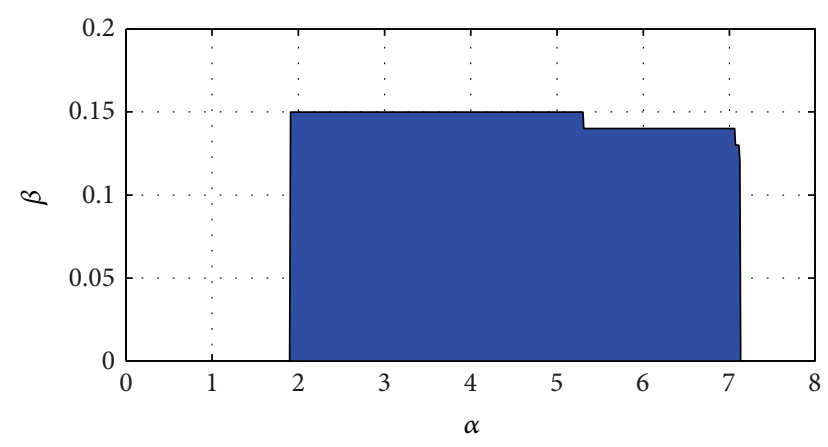

FIgURE 4: A region by $\alpha$ and $\beta$.

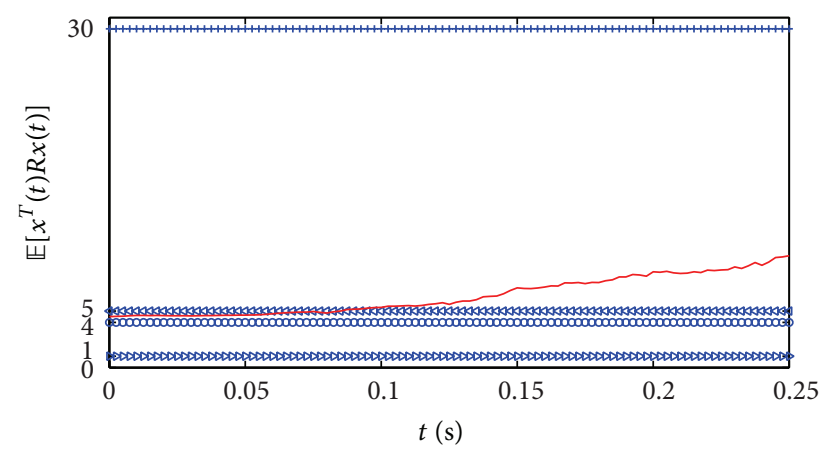

Figure 5: The evolution of $\mathbb{E}\left[x^{T}(t) R x(t)\right]$ of the closed-loop system of (1) in Case 2.

the feedback controller $u(t)=K \hat{x}(t)$ is chosen. Applying Algorithm 16 to Theorem 14, we obtain a region surrounded by $\alpha$ and $\beta$, which is illustrated by Figure 4 . By Figure 4 , selecting $\alpha=4, \beta=0$, and solving (57)-(63), we obtain

$$
\begin{aligned}
& \lambda_{1}=181.9, \quad \lambda_{2}=74.9, \quad \lambda_{3}=238.8, \quad \lambda_{4}=96.3 \text {, } \\
& \lambda_{5}=245.7, \quad \lambda_{6}=129.7, \quad \lambda_{7}=369.1 \text {, } \\
& Q_{1}=\left[\begin{array}{cc}
140.6 & -16.4 \\
-16.4 & 120.2
\end{array}\right], \quad Q_{2}=\left[\begin{array}{cc}
27.1 & -28.9 \\
-28.9 & 34.2
\end{array}\right], \\
& Q_{3}=\left[\begin{array}{cc}
111.4 & -13.3 \\
-13.3 & 92.6
\end{array}\right], \quad Q_{4}=\left[\begin{array}{cc}
207.1 & -42.9 \\
-42.9 & -158.4
\end{array}\right] \text {, } \\
& Q_{5}=\left[\begin{array}{cc}
331.6 & 62.9 \\
62.9 & 105.1
\end{array}\right], \quad N=\left[\begin{array}{ll}
-31.7 & 91.9
\end{array}\right]^{T} \text {. }
\end{aligned}
$$

Hence, the observer gain matrix is given by

$$
L=\widetilde{Q}_{2}^{-1} N=\left[\begin{array}{ll}
17.01 & 17.06
\end{array}\right]^{T} .
$$

The evolution of $\mathbb{E}\left[x^{T}(t) R x(t)\right]$ of the closed-loop system of (1) is illustrated by Figure 5, which shows that the closedloop system of (1) is finite-time stochastically bounded with respect to $(1,30,4,5,0.25, I, \mathscr{W})$. The corresponding control curves are illustrated by Figure 6 . Figure 7 illustrates the evolution of $\mathbb{E}\left[e^{T}(t) R e(t)\right]$ of the error system (54), which shows that $\mathbb{E}\left[e^{T}(t) \operatorname{Re}(t)\right]<1$.

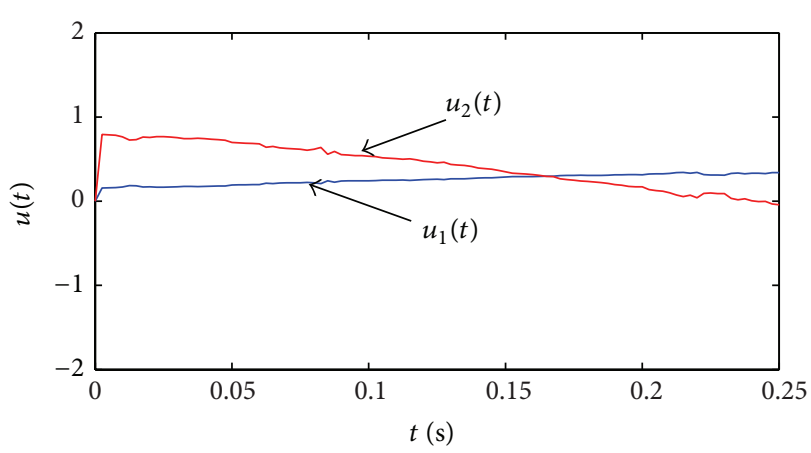

FIgURE 6: The evolution of $u(t)$.

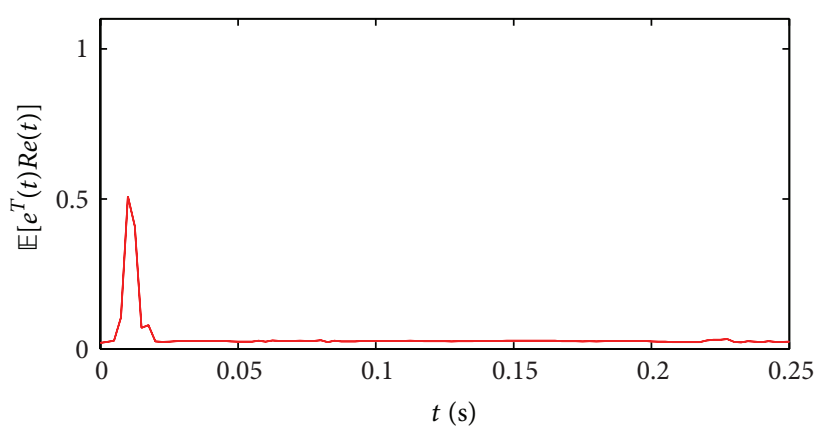

Figure 7: The evolution of $\mathbb{E}\left[e^{T}(t) \operatorname{Re}(t)\right]$.

\section{Conclusion}

In this study, finite-time stochastically bounded control linear stochastic Itô systems with $(x, u, v)$-dependent noise has been investigated. Applying different quadratic function approach, state and output feedback finite-time bounded controllers have been obtained, respectively. One example is presented to illustrate the effectiveness of the proposed results. In addition, we can also refer to [17-19] and extend the results of this paper to Takagi-Sugeno fuzzy systems, networked systems, linear parameter varying systems, and so on.

\section{Conflict of Interests}

The authors declare that there is no conflict of interests regarding the publication of this paper.

\section{Acknowledgments}

This work is supported by the Starting Research Foundation of Qilu University of Technology (Grant no. 12045501), the National Basic Research Program of China (973 Program) (Grant no. 2012CB215203), the National Natural Science Foundation of China (nos. 51036002 and 61203043). 


\section{References}

[1] F. Amato, M. Ariola, and P. Dorato, "Finite-time control of linear systems subject to parametric uncertainties and disturbances," Automatica, vol. 37, no. 9, pp. 1459-1463, 2001.

[2] F. Amato, M. Ariola, and C. Cosentino, "Finite-time stabilization via dynamic output feedback," Automatica, vol. 42, no. 2 , pp. 337-342, 2006.

[3] F. Amato and M. Ariola, "Finite-time control of discrete-time linear systems," IEEE Transactions on Automatic Control, vol. 50, no. 5, pp. 724-729, 2005.

[4] J.-E. Feng, Z. Wu, and J.-B. Sun, "Finite-time control of linear singular systems with parametric uncertainties and disturbances," Acta Automatica Sinica, vol. 31, no. 4, pp. 634-637, 2005.

[5] S. Zhao, J. Sun, and L. Liu, "Finite-time stability of linear timevarying singular systems with impulsive effects," International Journal of Control, vol. 81, no. 11, pp. 1824-1829, 2008.

[6] W. Zhang and X. An, "Finite-time control of linear stochastic systems," International Journal of Innovative Computing, Information and Control, vol. 4, no. 3, pp. 689-696, 2008.

[7] Z.-G. Yan and G.-S. Zhang, "Finite-time $H_{\infty}$ control for linear stochastic systems," Control and Decision, vol. 26, no. 8, pp. 1224-1228, 2011.

[8] S. He and F. Liu, "Stochastic finite-time stabilization for uncertain jump systems via state feedback," Journal of Dynamic Systems, Measurement and Control, vol. 132, no. 3, Article ID 034504, 4 pages, 2010.

[9] Y. Zhang, C. Liu, and X. Mu, "Robust finite-time $H_{\infty}$ control of singular stochastic systems via static output feedback," Applied Mathematics and Computation, vol. 218, no. 9, pp. 5629-5640, 2012.

[10] Y. Yang, J. Li, and G. Chen, "Finite-time stability and stabilization of nonlinear stochastic hybrid systems," Journal of Mathematical Analysis and Applications, vol. 356, no. 1, pp. 338345, 2009.

[11] Z. Yan, G. Zhang, and J. Wang, "Non-fragile robust finitetime $H_{\infty}$ control for nonlinear stochastic Itô systems using neural network," International Journal of Control, Automation and Systems, vol. 10, no. 5, pp. 873-882, 2012.

[12] Z.-G. Yan and G.-S. Zhang, "Finite-time $H_{\infty}$ filtering for a class of nonlinear stochastic uncertain systems," Control and Decision, vol. 27, no. 3, pp. 419-424, 2012.

[13] F. Amato, R. Ambrosino, M. Ariola, C. Cosentino, and G. D. Tommasi, Finite-Time Stability and Control, vol. 453 of Lecture Notes in Control and Information Sciences, Springer, London, UK, 2014

[14] Z. Yan, G. Zhang, and W. Zhang, "Finite-time stability and stabilization of linear Itô stochastic systems with state and control-dependent noise," Asian Journal of Control, vol. 15, no. 1, pp. 270-281, 2013.

[15] W. Zhang, H. Zhang, and B.-S. Chen, "Stochastic $H_{2} / H_{\infty}$ control with $(x, u, v)$-dependent noise: finite horizon case," Automatica, vol. 42, no. 11, pp. 1891-1898, 2006.

[16] W. Zhang and G. Feng, "Nonlinear stochastic $H_{2} / H_{\infty}$ control with $(x, u, v)$-dependent noise: infinite horizon case," IEEE Transactions on Automatic Control, vol. 53, no. 5, pp. 1323-1328, 2008.

[17] H. Zhang, Y. Shi, and B. X. Mu, "Optimal $H_{\infty}$-based linearquadratic regulator tracking control for discrete-time TakagiSugeno fuzzy systems with preview actions," Journal of Dynamic Systems, Measurement and Control, vol. 135, no. 4, Article ID 044501, 5 pages, 2013.
[18] H. Zhang, Y. Shi, and A. S. Mehr, "Robust $H_{\infty}$ PID control for multivariable networked control systems with disturbance/noise attenuation," International Journal of Robust and Nonlinear Control, vol. 22, no. 2, pp. 183-204, 2012.

[19] H. Zhang and Y. Shi, "Parameter-dependent $H_{\infty}$ filtering for linear parameter varying systems," Journal of Dynamic Systems, Measurement and Control, vol. 135, no. 2, Article ID 021006, 7 pages, 2012. 


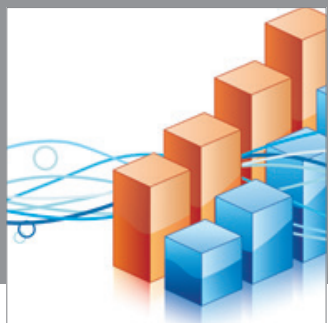

Advances in

Operations Research

mansans

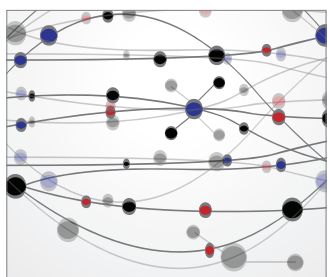

The Scientific World Journal
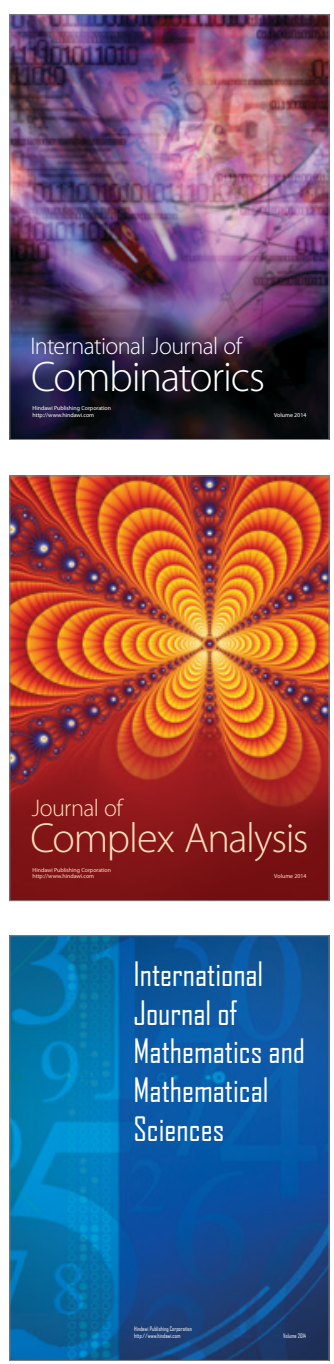
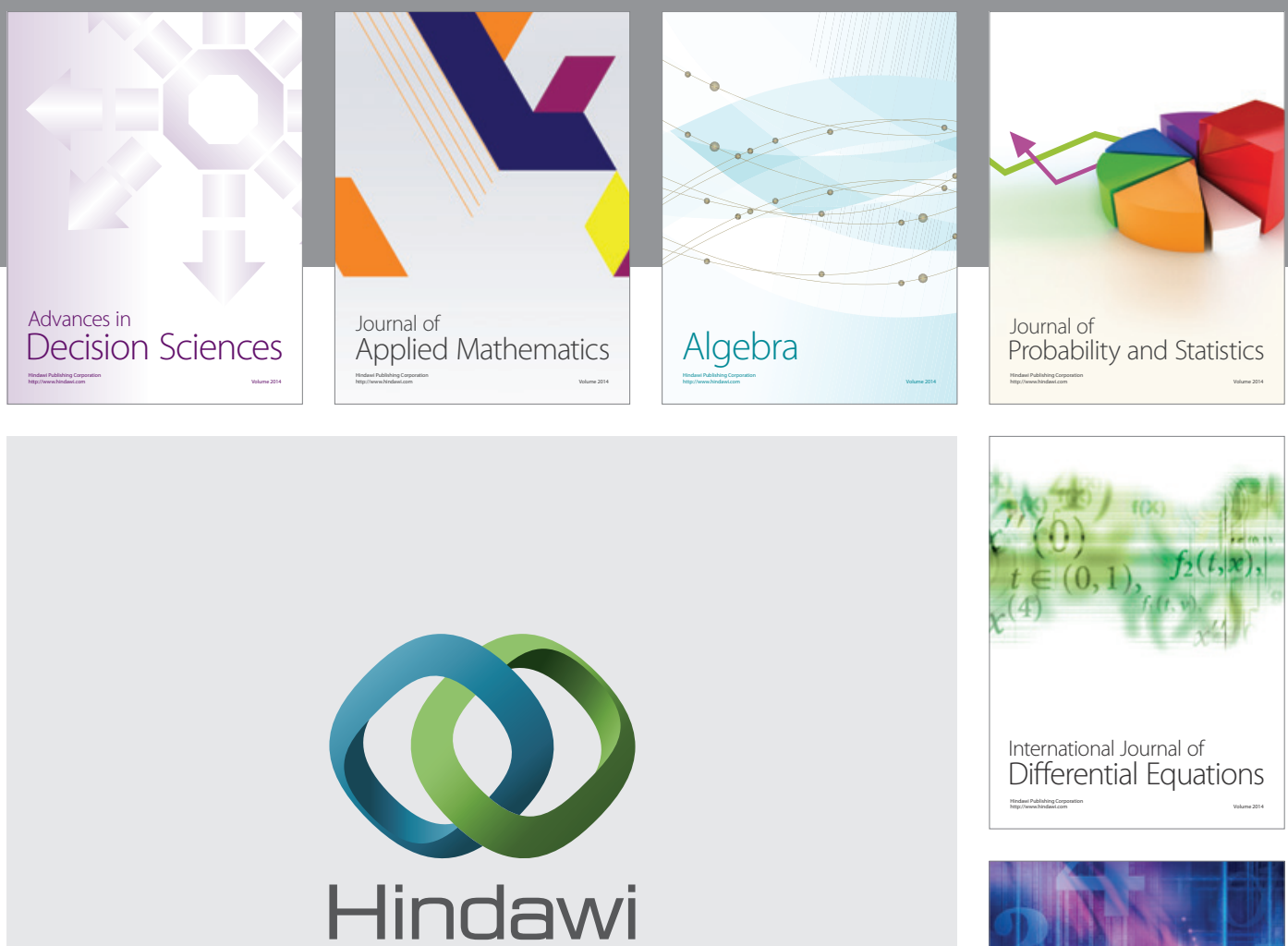

Submit your manuscripts at http://www.hindawi.com
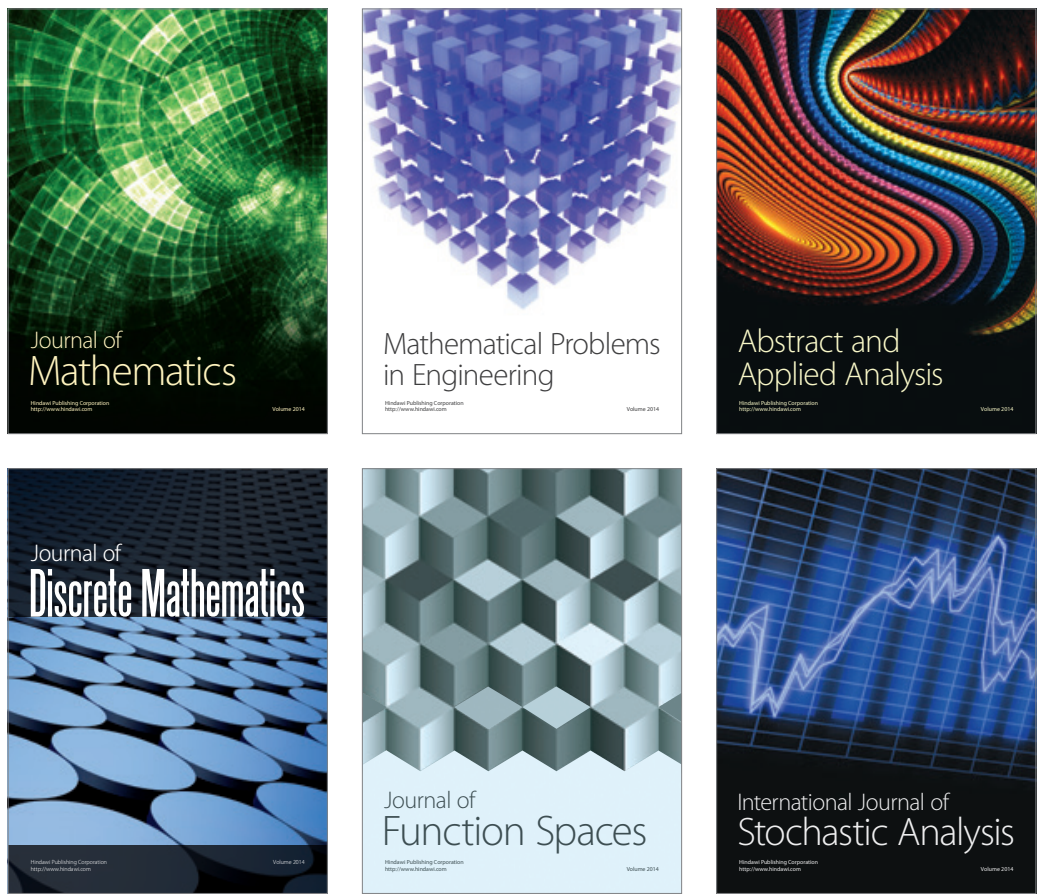

Journal of

Function Spaces

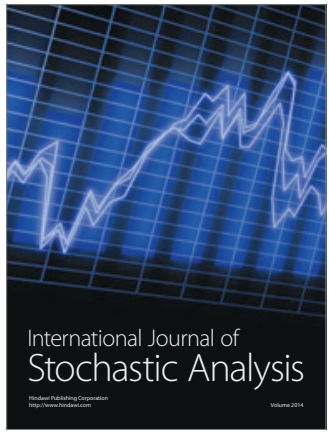

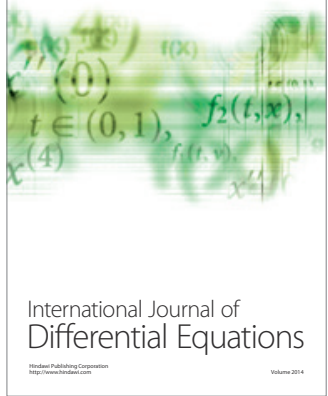
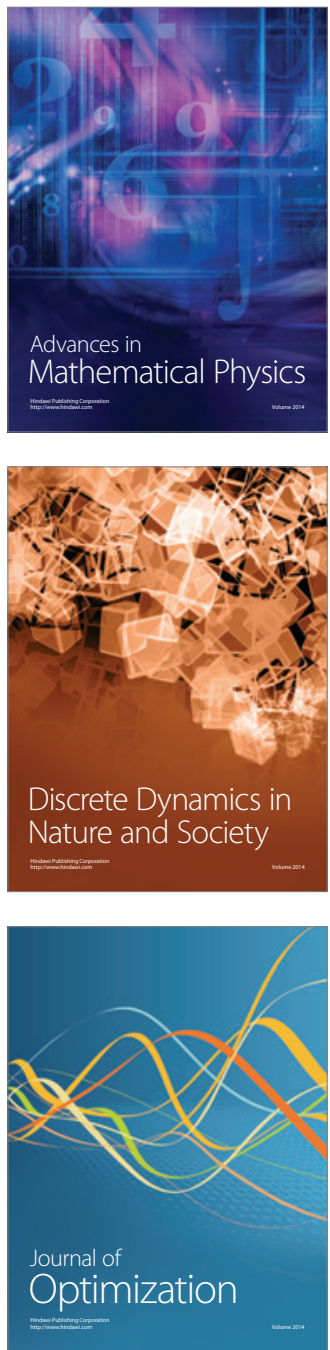